\title{
FIXED POINTS OF ORDER PRESERVING MULTIFUNCTIONS
}

\author{
R. E. SMITHSON
}

Abstract. Let $F: X \rightarrow X$ be a multifunction on a partially ordered set $(X, \leqq)$. Suppose for each pair $x_{1} \leqq x_{2}$ and for each $y_{1} \in F\left(x_{1}\right)$ there is a $y_{2} \in F\left(y_{2}\right)$ such that $y_{1} \leqq y_{2}$. Then sufficient conditions are given such that multifunctions $F$ satisfying the above condition will have a fixed point. These results generalize the Tarski Theorem on complete lattices, and they also generalize some results of S. Abian and A. B. Brown, Canad. J. Math 13 (1961), 78-82. By similar techniques two selection theorems are obtained. Further, some related results on quasi-ordered and partially ordered topological spaces are proved. In particular, a fixed point theorem for order preserving multifunctions on a class of partially ordered topological spaces is obtained.

1. Multifunctions on partially ordered sets. Tarski's result on the existence of a fixed point for an isotone function on a complete lattice is well known (see Birkhoff [3, p. 115, Theorem 11]), and a number of related results have also been published. For example, S. Abian and A. B. Brown [2] published results on nondecreasing maps on a partially ordered set, and A. Abian [1] obtained a result for nonincreasing functions on a totally ordered set. Then in 1954, L. E. Ward, Jr. [6] published several results for continuous order preserving functions on quasi-ordered and partially ordered topological spaces. The purpose of the present paper is to present results analogous to these for multivalued functions.

In this paper a multifunction $F: X \rightarrow Y$ is a correspondence such that $\varnothing \neq F(x) \subset Y$ for each $x \in X$ where $\varnothing$ is the empty set. Multifunctions will be denoted by $F, G$, etc. Let $P$ be a property of sets. Then a multifunction $F$ is said to be point $P$ in case $F(x)$ has property $P$ for each $x$ in the domain. Finally a fixed point of $F$ is a point $x$ such that $x \in F(x)$.

Let $F: X \rightarrow Y$ and let $\leqq$ denote a relation on $X$ and a relation on $Y$. Then we shall use the following two conditions throughout this paper.

Received by the editors March 13, 1970.

AMS 1969 subject classifications. Primary 9620, 5485, 5465; Secondary 0630, 5456.

Key words and phrases. Partially ordered sets, fixed point theorems on partially ordered sets, multivalued functions on partially ordered sets, order preserving functions, fixed points for order preserving multivalued functions, partially ordered topological spaces, fixed points of order preserving multifunctions on partially ordered topological spaces. 
I. If $x_{1} \leqq x_{2} \in X$, and if $y_{1} \in F\left(x_{1}\right)$, then there is $y_{2} \in F\left(x_{2}\right)$ such that $y_{1} \leqq y_{2}$.

II. If $x_{1} \leqq x_{2} \in X$ and if $y_{2} \in F\left(x_{2}\right)$, then there is $y_{1} \in F\left(x_{1}\right)$ such that $y_{1} \leqq y_{2}$. Simple examples show that one of these conditions may be satisfied while the other is not.

In the following, the terms least upper bound (lub), greatest lower bound (glb), chain, etc. will be used in their usual sense. Also, an isotone map is a function $f$ such that $x_{1} \leqq x_{2}$ implies that $f\left(x_{1}\right) \leqq f\left(x_{2}\right)$. For our first result we shall use the following condition.

III. Let $F: X \rightarrow X$ and let $C$ be a chain in $X$. Suppose that there is an isotone function $g: C \rightarrow X$ such that $g(x) \in F(x)$ for all $x \in C$. If $x_{0}=\operatorname{lub} C$, then there exist $y_{0} \in F\left(x_{0}\right)$ such that $g(x) \leqq y_{0}$ for all $x \in C$.

TheOREM 1.1. Let $X$ be a partially ordered set in which each nonempty chain has a least upper bound, and suppose $F$ is a multifunction on $F$ which satisfies conditions I and III. If there is a point $e \in X$ and $a$ point $y \in F(e)$ such that $e \leqq y$, then $F$ has a fixed point.

Proof. Let $e \in X$ and let $y \in F(e)$ with $e \leqq y$. Define a collection $\delta$ of subsets $Y$ of $X$ by:

(1) $e \in Y$,

(2) if $e \leqq z \leqq x \in Y$, then $z \in Y$,

(3) if $x \in Y$, then there is a $z \in F(x)$ such that $x \leqq z$.

Partial order $S$ by inclusion. Note that $\{e\} \in \mathcal{S}$ and hence, by Zorn's Lemma there is a maximal element $X_{0} \in \delta$. Now let $C$ be a maximal chain in $X_{0}$ ( $C$ exists by the Hausdorff maximal principle), and let $x_{0}=\operatorname{lub} C$.

We now show that $x_{0} \in X_{0}$. For this we claim that there is a subset $C_{0} \subset C$ such that (i) $x_{0}=$ lub $C_{0}$ and (ii) there is an isotone function $g: C_{0} \rightarrow X$ such that $g(x) \in F(x)$ and $x \leqq g(x)$ for each $x \in C_{0}$. Let $\mathfrak{e}$ be the collection of subsets of $C$ which satisfy (ii). If $C_{1}, C_{2} \in \mathbb{e}$, then partial order the pairs $\left(C_{1}, g_{1}\right),\left(C_{2}, g_{2}\right)$ where $g_{i}$ is a fixed function from condition (ii) by $\left(C_{1}, g_{1}\right) \prec\left(C_{2}, g_{2}\right)$ iff $C_{1} \subset C_{2}$ and $g_{1}=g_{2} \mid C_{1}$. Then by Zorn's Lemma there is a maximal set $C_{0}$ with function $g_{0}$ in $\mathcal{C}$. Let $x^{\prime}=$ lub $C_{0}$. If $x^{\prime} \neq x_{0}$, then there is an $x \in C$ such that $x^{\prime}<x$. Further by using conditions I and III we can extend $g_{0}$ to the set $C_{0} \cup\{x\}$, and contradict the maximality of $C_{0}$. Thus lub $C_{0}=x_{0}$. Finally by condition III, there is a $y \in F\left(x_{0}\right)$ such that $g(x) \leqq y$ for all $x \in C_{0}$. But $x \leqq g(x)$ for $x \in C_{0}$ and so $y$ is an upper bound for $C_{0}$. Hence, $x_{0} \leqq y$ and $x_{0} \in X_{0}$.

Finally, since $x_{0} \in X_{0}$, there exists a $y_{0} \in F\left(x_{0}\right)$ such that $x_{0} \leqq y_{0}$. If $x_{0}=y_{0}$, we are done. Thus suppose $x_{0}<y_{0}$. Then set 


$$
X_{1}=X_{0} \cup\left\{z: x_{0} \leqq z \leqq y_{0}\right\}
$$

By condition $I$ for each $z, x_{0} \leqq z \leqq y_{0}$, there is a $w \in F(z)$ such that $y_{0} \leqq w$. But then $z \leqq w$. Thus (1), (2), and (3) are satisfied by $X_{1}$. This contradicts the maximality of $X_{0}$, and thus $x_{0} \in F\left(x_{0}\right)$.

Since every isotone single valued function satisfies conditions I and III we have the following corollary.

COROLlaRY 1.2. Let $X$ be a partially ordered set in which each chain has a least upper bound. If $F: X \rightarrow X$ is an isotone function on $X_{1}$ and if there is an $e \in X$ such that $e \leqq f(e)$, then $f$ has a fixed point.

In 1955 Anne C. Davis [4] characterized complete lattices in terms of the fixed point property for increasing functions. By combining her result with Theorem 1.1 we obtain the following corollary.

Corollary 1.3. Let $X$ be a lattice. Then $X$ is complete if and only if every multifunction on $X$ which satisfies conditions I and III has a fixed point.

In 1957 Ward [7] extended Davis' [4] result to semilattices. By combining the techniques used in proving Theorem 1.1 with the techniques used by Ward in [7] we obtain the semilattice analog of Theorem 1.1. Then we obtain Corollary $1.3^{\prime}$ by applying this remark and Ward's results. The nomenclature of semilattices used in Corollary $1.3^{\prime}$ is that of Ward [7].

Corollary $1.3^{\prime}$. Let $(X, \leqq)$ be a semilattice. Then $(X, \leqq)$ is compact in its interval topology if and only if each multifunction on $X$ into $X$ which satisfies conditions I and III has a fixed point.

REMARK. By suitably altering the hypothesis and by giving a version of condition III using glb's rather than lub's we can obtain analogs of the above results with condition II in place of condition I.

It first appears that Corollary 1.2 is weaker than Theorem 2 of [2]. However, the following lemma shows that this is not the case, and that Theorem 2 in [2] is in fact a corollary of Theorem 1.1. This lemma or some form of it seems to be a part of the folklore. In fact, the essential part of the proof-that every chain contains a wellordered cofinal subset-appears as an exercise in [3] and in Halmos' Naive set theory. For this reason the proof of Lemma 1.4 is omitted.

LEMMA 1.4. In a partially ordered set $X$ the following are equivalent.

(1) Every nonempty well-ordered set in $X$ has a lub in $X$.

(2) Every nonempty chain in $X$ has a lub in $X$. 
Corollary 1.5 (ABian ANd Brown). Let $X$ be a partially ordered set in which every nonempty well-ordered set has a lub in $X$. Let $f: X \rightarrow X$ be an isotone function of $X$. If there is a point $e \in X$ such that $e \leqq f(e)$, then f has a fixed point.

Another proposition which is an immediate consequence of Lemma 1.4 is the following multifunction version of Abian and Brown's Theorem 3.

Proposition 1.6. Let $X$ be a partially ordered set in which every nonempty well-ordered set has a lub in $X$. Let $F: X \rightarrow X$ be a multifunction on $X$ such that for each $x \in X$ there exists $a y \in F(x)$ such that $x \leqq y$. Then $F$ has a fixed point.

Proof. Apply the Hausdorff maximal principle to obtain a maximal chain. By Lemma 1.4 this chain must have a lub in $X$ which is necessarily a fixed point.

Now we give a simple example which shows the importance of condition III. Let $X=[0,1]$, and define $F: X \rightarrow X$ by $F(x)=(x+1) / 2$ for $x \in[0,1)$, and $F(1)=[0,1)$. Then condition $I$ is satisfied, but condition III is not satisfied. Furthermore, $F$ does not have a fixed point.

We conclude this section with two selection theorems for multifunctions on partially ordered sets.

Definition. Let $F: X \rightarrow Y$ be a multifunction on $X$ into $Y$. A selection for $F$ is a single valued function $f: X \rightarrow Y$ such that $f(x) \in F(x)$ for each $x \in X$. An isotone (continuous, etc.) selection is a selection which is isotone (continuous, etc.).

Theorem 1.7. Let $F$ be a partially ordered set, and let $F: X \rightarrow X$ be a multifunction on $X$ which satisfies condition I. If lub $F(x) \in F(x)$ for all $x \in X$, then there is an isotone selection for $F$.

PROof. Let $f(x)=\operatorname{lub} F(x)$ for each $x \in X$. Then $f$ is a selection for $F$. To see that $f$ is isotone let $x_{1} \leqq x_{2}$. Since $f\left(x_{1}\right) \in F\left(x_{1}\right)$, there is a $z \in F\left(x_{2}\right)$ (by condition I) such that $f\left(x_{1}\right) \leqq z$. But $z \leqq \operatorname{lub} F\left(x_{2}\right)=f\left(x_{2}\right)$, and so $f\left(x_{1}\right) \leqq f\left(x_{2}\right)$.

A similar result could be obtained by using the greatest lower bound of the sets $F(x)$ and condition II. As above we obtain a corollary to Theorem 1.7 analogous to Corollary 1.3.

Corollary 1.8. Let $X$ be a lattice and let $F: X \rightarrow X$ be a multifunction on $X$. Then $X$ is complete if and only if whenever $F$ satisfies condition I (II) and whenever lub $F(x) \in F(x)$ (glb $F(x) \in F(x))$ for all $x \in X, F$ has a fixed point. 
For the last result in this section we need the following conditions.

IV. Let $x_{1} \leqq x_{2}$ and let $y_{1} \in F\left(x_{1}\right), y_{2} \in F\left(x_{2}\right)$ with $y_{1} \leqq y_{2}$. If $x_{1}$ $\leqq x \leqq x_{2}$, then $F(x) \cap\left\{z: y_{1} \leqq z \leqq y_{2}\right\} \neq \varnothing$.

V. If $C=\left\{z: x_{1} \leqq z \leqq x_{2}\right\}$ for $x_{1} \leqq x_{2}$, and if $F(x) \cap C \neq \varnothing$, then $\operatorname{lub}(C \cap F(x)) \in C \cap F(x)$.

TheOREM 1.9. Let $X$ be a partially ordered set which contains a least element $e$ and in which each chain has a least upper bound in $X$. If $F: X \rightarrow X$ is a multifunction on $X$ which satisfies conditions III, IV and $\mathrm{V}$, then there is an isotone selection $F$ on $X$.

Proof. Let $s$ be the collection of subsets $Y$ of $X$ such that (1) $e \in Y$, (2) if $z \leqq x \in Y$, then $z \in Y$, and (3) there is an isotone function $g: Y \rightarrow X$ such that $g(x) \in F(x)$ for all $x \in Y$. We partial order the pairs $(Y, g)$ where $Y \in S$ and $g$ is a fixed function from (3) as follows: $\left(Y_{1}, g_{1}\right) \prec\left(Y_{2}, g_{2}\right)$ iff $Y_{1} \subset Y_{2}$ and $g_{1}=g_{2} \mid Y_{1}$. Then by Zorn's Lemma there is a maximal pair $\left(X_{0}, f_{0}\right)$. If $X=X_{0}$, we are done; thus suppose that $x \in X \backslash X_{0}$, and let $C$ be a maximal chain containing $e$ and $x$. Then $C \cap X_{0}=C_{1}$ is a chain in $X_{0}$. Let $x_{0}=$ lub $C_{1}$. By applying condition III and the maximality of $X$ we deduce that $x_{0} \in X_{0}$. Now pick a $y^{\prime} \in F(x)$ such that $f_{0}\left(x_{0}\right) \leqq y^{\prime}$. Then set $Y=X_{0} \cup\left\{z: x_{0} \leqq z \leqq x\right\}$ and define $f$ on $Y$ as follows. If $z \in X_{0}$, then $f(z)=f_{0}(z)$. If $x_{0}<z<x$, then $f(z)=$ lub $\left(F(z) \cap\left\{y: f_{0}\left(x_{0}\right) \leqq y \leqq y^{\prime}\right\}\right)$ and set $f(x)=y^{\prime}$. Conditions IV and $\mathrm{V}$ show that $f$ is well defined and isotone. But this contradicts the maximality of $X_{0}$. Hence, the theorem follows.

2. Partially ordered and quasi-ordered topological spaces. Ward's generalizations of the fixed point theorem for order preserving functions were concerned with order preserving continuous functions on partially ordered topological spaces. In the remaining portion of this paper we show that similar results are true for certain classes of multifunctions. For this purpose we present the following definitions and conventions which are from Ward [6].

Let $X$ be a set. Then a quasi-order, $\leqq$, is a reflexive, transitive relation on $X$. Further, if a quasi-order $\leqq$ is antisymmetric then it is a partial order. A chain is a linearly ordered subset of $X$; if $\leqq$ is a quasi-order, and if $x \in X$, then $E(x)=\{z: z \leqq x$ and $x \leqq z\}$. Set $L(x)$ $=\{z: z \leqq x\}$, and $M(x)=\{z: x \leqq z\}$. Now if $X$ is a topological space with a quasi-order $\leqq$, then $X$ is called a quasi-ordered topological space (Q.O.T.S.) in case $L(x)$ and $M(x)$ are closed sets for each $x \in X$, and, if $\leqq$ is a partial order, then $X$ is a partially ordered topological space (P.O.T.S.). Further, $\leqq$ is continuous in case the relation $\leqq$ is a closed subset of $X \times X$. (For a discussion of these concepts and 
their consequences see Ward [6] and also Nachbin [5].) Finally, in the following, $X$ will denote a Hausdorff topological space.

LEMma 2.1. If $X$ is a compact Q.O.T.S. with continuous quasi-order, then every monotone net in $X$ clusters, and the set of cluster points is contained in $E\left(x_{0}\right)$, for some $x_{0} \in X$.

The proof of Lemma 2.1 is in [5] and is omitted here.

Corollary. If $X$ is a compact P.O.T.S. with continuous order, then every monotone net in $X$ converges.

Definition. A multifunction $F: X \rightarrow Y$ on the topological space $X$ into the space $Y$ is upper semicontinuous (u.s.c.) iff $F^{-1}(A)$ is closed for each closed subset $A \subset Y$.

The following lemma is an extension of Lemma 6 in [6].

Lemma 2.2. Let $F: X \rightarrow X$ be an u.s.c. point compact multifunction and let $S$ be a sequence in $Y$ such that $x_{n} \in F\left(x_{n-1}\right)$ for $n \geqq 1$. If $z$ is a cluster point of $S$, then there is a point in $F(z)$ which is a cluster point of $S$.

Proof. Suppose not. Then, since $F(z)$ is compact, we can construct an open set $U$ such that $F(z) \subset U$ and $x_{n} \notin U$ for all but a finite number of values of $n \geqq 0$. But if $x_{n_{i}} \rightarrow z$, then since $F$ is u.s.c., there is an $i$ such that $F\left(x_{n_{j}}\right) \subset U$ for $j>i$. This is a contradiction. Hence, some point of $F(z)$ is a cluster point of $s$.

Before stating the next theorem we need the following definition.

Definition. A subset $A$ of a quasi-ordered set $X$ is weakly convex iff $a, b \in A$ and $a \leqq x \leqq b$ implies that $E(x) \cap A \neq \varnothing$.

TheOREM 2.3. Let $X$ be a Q.O.T.S. in which the maximal chains are compact, and let $F: X \rightarrow X$ be a point compact, u.s.c. multifunction which satisfies conditions I and II above, and such that $F(x)$ is weakly convex for each $x \in X$. Then a necessary and sufficient condition that there exist a nonempty compact set $K \subset E\left(x_{0}\right)$ for some $x_{0} \in X$, such that $F(x) \cap K$ $\neq \varnothing$ for each $x \in K$ is that there exist an $x \in X$ such that for some $y \in F(x)$ either $y \leqq x$ or $x \leqq y$ (i.e. $x$ is comparable to some point in $F(x)$ ).

Proof. The necessity is clear. Thus suppose there is an $x_{0}$ which is comparable to some point in $F\left(x_{0}\right)$. Then by applying either condition I or II, we can construct a monotone sequence $\delta$ such that $x_{n} \in F\left(x_{n-1}\right), n \geqq 1$. Then the set $\left\{x_{n}: n \geqq 0\right\}$ is contained in a maximal chain which by hypothesis is compact. Furthermore, for linear orders, continuity and semicontinuity of the quasi-order are equivalent. Hence, we can apply Lemma 2.1 and obtain a cluster point $z_{0}$ of $s$. Moreover, each cluster point of $s$ is contained in $E\left(z_{0}\right)$. 
Now if $: \in E\left(z_{0}\right), z \leqq z_{0}$ and $z_{0} \leqq z$. There is a $w \in F\left(z_{0}\right)$ which is a cluster point of $S$ by Lemma 2.2, and by Lemma 2.1, $w \in E\left(x_{0}\right)$. Thus by conditions I and II there are $y_{1}, y_{2} \in F(z)$ such that $y_{1} \leqq w \leqq y_{2}$, and hence, since $F(z)$ is weakly convex, $F(z) \cap E\left(z_{0}\right) \neq \varnothing$.

Observe that $E\left(z_{0}\right)$ is a chain and is the intersection of closed sets. Thus $E\left(z_{0}\right)$ is compact. Now define $K_{1}=F\left(E\left(z_{0}\right)\right) \cap E\left(z_{0}\right)$, and inductively $K_{n}=F\left(K_{n-1}\right) \cap E\left(z_{0}\right)$ for $n>1$. Then set $K=\bigcap_{n=1}^{\infty} K_{n}$. Clearly $K$ is a nonempty compact subset of $E\left(z_{0}\right)$. (Note that an u.s.c. point compact multifunction preserves compact sets.)

Finally, let $z \in K$. Then $F(z) \cap K_{n}$ is nonempty for each $n$, and since $K_{n} \subset K_{n-1}$, the set $\left\{F(z) \cap K_{n}: n \geqq 1\right\}$ has f.i.p. (finite interpolation property). Thus there is a $y \in \bigcap\left\{F(z) \cap K_{n}: n \geqq 1\right\}$, and hence, $F(z) \cap K \neq \varnothing$.

COROLlaRY. If, in addition $X$ is partially ordered, then a necessary and sufficient condition that $F$ have a fixed point is that there exist an $x \in X$ which is comparable to some point of $F(x)$.

\section{REFERENCES}

1. A. Abian, $A$ fixed point theorem for nonincreasing mappings, Boll. Un. Mat. Ital. (4) 2 (1969), 200-201. MR 39 \#5427.

2. S. Abian and A. B. Brown, $A$ theorem on partially ordered sets, with applications to fixed point theorems, Canad. J. Math. 13 (1961), 78-82. MR 23 \#A817.

3. G. Birkhoff, Lattice theory, 3rd ed., Amer. Math. Soc. Colloq. Publ., vol. 25, Amer. Math. Soc., Providence, R. I., 1967. MR 37 \#2638.

4. A. C. Davis (A. C. Morel), A characterization of complete lattices, Pacific J. Math. 5 (1955), 311-319. MR 17, 574.

5. L. Nachbin, Topology and order, Van Nostrand, Princeton, N. J., 1965. MR 36 \#2125.

6. L. E. Ward, Partially ordered topological spaces, Proc. Amer. Math. Soc. 5 (1954), 144-161. MR 16, 59.

7. —- Completeness in semi-lattices, Canad. J. Math. 9 (1957), 578-582. MR 19, 938 .

UNIVERSity OF Wyoming, LARAMIE, Wyoming 82070 